\title{
Short communication: Bovine-derived proteins activate STAT3 in human skeletal muscle in vitro
}

\author{
M. K. Caldow, ${ }^{*} \dagger^{1}$ M. R. Digby, $\ddagger$ and D. Cameron-Smith ${ }^{\star} \S$ \\ *Molecular Nutrition Unit, School of Exercise and Nutrition Sciences, Deakin University, Melbourne, 3125, Australia \\ †Basic and Clinical Myology Laboratory, Department of Physiology, University of Melbourne, Melbourne, 3010, Australia \\ łDepartment of Zoology, University of Melbourne, Melbourne, 3010, Australia \\ §Liggins Institute, University of Auckland, Auckland 1142, New Zealand
}

\begin{abstract}
Bovine milk contains biologically active peptides that may modulate growth and development within humans. In this study, targeted bovine-derived proteins were evaluated for their effects on signal transducer and activator of transcription-3 (STAT3) phosphorylation in human skeletal muscle cells. Following an acute exposure, bovine-derived acidic fibroblast growth factor-1 (FGF) and leukemia inhibitory factor (LIF) activated STAT3 in differentiating myotubes. Chronic exposure to FGF and LIF during the proliferative phase reduced myoblast proliferation and elevated MyoD and creatine kinase (CKM) mRNA expression without altering apoptotic genes. In mature myotubes, neither FGF nor LIF elicited any action. Together, these data indicate that a reduction in proliferation in the presence of bovine-derived FGF or LIF may stimulate early maturation of myoblasts.
\end{abstract}

Key words: bovine protein, skeletal muscle, STAT3, growth factor, cytokine

\section{Short Communication}

Milk and milk products contain biologically active proteins that contribute in largely undefined mechanisms to optimal growth, development, and nutrition (Séverin and Wenshui, 2005). These proteins include cytokines, chemokines (Garofalo and Goldman, 1998), and growth factors (Grosvenor et al., 1993). Bovine colostrum, whey, and whole milk have been used in place of serum, with increased cell proliferation observed in a variety of cells of rodent origin (Steimer et al., 1981). Whey fractions can also be used as a supplement for cell culture medium to maintain long-term growth of cells (Damerdji et al., 1988; Belford et al., 1995), and promote wound repair in a wounded-skin culture model (Rayner et al., 2000).

Received October 28, 2014.

Accepted January 14, 2015.

${ }^{1}$ Corresponding author: marissa.caldow@unimelb.edu.au
Few studies address both the proteins responsible for the cell action and further the cellular responses in the largest tissue, skeletal muscle. Numerous intracellular signaling pathways play critical roles in myogenesis and regeneration. The Janus kinase/signal transducer and activator of transcription (JAK/STAT) pathway, regulated by an array of cytokines and growth factors, orchestrates the development of a range of tissues. Signal transducer and activator of transcription-3 (STAT3) is an integral component of the proliferation/ differentiation process in skeletal muscle in response to IL-6 and leukemia inhibitory factor (LIF; Brown and Blakeley, 1984; Sun et al., 2007). Dairy proteins have been proposed to promote skeletal muscle protein synthesis and aid in muscle recovery following resistance exercise (see review by Crittenden et al., 2009). Yet it is currently unknown if bovine-derived proteins are capable of activating JAK/STAT signaling within human skeletal muscle. Thus, the aim of this study was to evaluate the ability of bovine-derived compounds to regulate myogenesis in human primary skeletal muscle cells in vitro. Proteins were chosen for their potential to activate STAT3 if they were either a direct activator of JAK/STAT in other cell models or related to a known mediator of the pathway. Of the 14 bovine-derived proteins screened, we identified 2 that were able to induce human STAT3 phosphorylation.

Products of PCR, encompassing the entire coding region, were produced from lactating cow mammary gland. Products were directly cloned into the mammalian expression vector pTarget (Promega, Madison, WI), under CMV promoter control. Clones were sequenced to verify sequence fidelity and orientation. The HEK293 cells supplied with the pTarget vector (Promega) were grown to $80 \%$ confluence in Dulbecco's modified Eagle's medium (DMEM; Life Technologies, Melbourne, Victoria, Australia). Following the manufacturer's instructions, cells were transfected with $4 \mu \mathrm{g}$ of plasmid DNA using Lipofectamine 2000 (Life Technologies) and incubated at $37^{\circ} \mathrm{C}$ for $4 \mathrm{~h}$. Transfection medium was removed and replaced with $\alpha$-Minimum Essential Medium ( $\boldsymbol{\alpha}$-MEM) containing 2\% horse 
serum (HS; Life Technologies), $0.05 \%$ penicillin/streptomycin (Life Technologies), and $0.05 \%$ amphotericin (Life Technologies). After a further $36 \mathrm{~h}$ of incubation, the conditioned medium was collected, debris removed by centrifugation, and the medium was stored frozen at $-80^{\circ} \mathrm{C}$ until used.

Skeletal muscle samples were taken from the vastus lateralis of healthy young males ( $24 \pm 2$ yr old) using the percutaneous needle biopsy technique (Bergstrom, 1975) modified to include suction (Evans et al., 1982), and primary human muscle cells were isolated and cultured as described previously (Caldow et al., 2011). All procedures performed were in accordance with the ethical standards of Deakin University and with the Helsinki Declaration of 1964 and its later amendments or comparable ethical standards.

Briefly, cells were induced to differentiate with

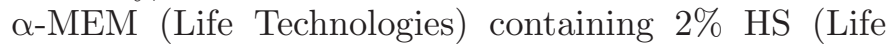
Technologies), $\quad 0.05 \%$ penicillin/streptomycin (Life Technologies), and $0.05 \%$ amphotericin (Life Technologies) for $4 \mathrm{~d}$. On d 4, cells were exposed to a 1:20 dilution of bovine-derived compounds/conditioned media: chemokine (C-C motif) ligand 28 (CCL28); chemokine (C-C motif) ligand 3 (CCL3); chemokine (C-C motif) ligand 3-like 1 (CCL3L1); acidic fibroblast growth factor (FGF); fibroblast growth factor binding protein 1 (FGFBP1); interferon a 21 (IFN 21 ); IGFI; LIF; nuclear receptor subfamily 1 , group $\mathrm{H}$, member 2 (NR1H2); neuregulin 1 (NRG1); placental growth factor (PGF); transmembrane protein induced by TNF- $\alpha$ (TMPIT); tumor necrosis factor ligand superfamily, member 13 (TNFSF13); and vascular endothelial growth factor (VEGF) in differentiation medium for 30 min. The concentration of expressed proteins in conditioned media may be as high as $1 \mu \mathrm{g} / \mathrm{mL}$, and may vary between specific proteins depending on size and stability. Expressed proteins in the final 1:20 dilution were at approximately $50 \mathrm{ng} / \mathrm{mL}$. Conditioned medium from cells exposed to an empty vector was used as the control. Cells treated with $10 \mathrm{ng} / \mathrm{mL}$ of IL-6 (Life Technologies) for 30 min were used as a positive control.

For experiments in proliferating cells, bovine-derived FGF or LIF was added to growth medium for $4 \mathrm{~d}$, with treatments refreshed daily. For differentiation experiments, cells were treated after $5 \mathrm{~d}$ of differentiation with FGF or LIF in differentiation medium for $4 \mathrm{~d}$, with treatments refreshed daily. An empty vector was used as the control.

Cells $\left(1.5 \times 10^{3}\right)$ were seeded onto extracellular matrix (ECM)-coated (Sigma-Aldrich, St. Louis, MO) 96-well plates and left for $48 \mathrm{~h}$. Cells were treated with FGF, LIF, empty vector (control), or growth medium alone for $4 \mathrm{~d}$, refreshing treatments daily. On d 4, following the manufacturer's instructions, cells were exposed to $20 \mu \mathrm{L}$ of CellTiter 96 AQueous One Solution Reagent for $3 \mathrm{~h}$ (Promega). Absorbance was measured at 490 $\mathrm{nm}$ on a BioTek Synergy 2 Multi-Mode Microplate Reader using Gen5 Data Analysis Software (BioTek Instruments, Millennium Science, Mulgrave, Australia).

Cells were collected and lysates were prepared for immunoblotting as previously described (Trenerry et al., 2011). Primary antibodies, phosphorylated (p) STAT3 and total (t)STAT3 (Cell Signaling, Danvers, MA) diluted in 5\% BSA (Sigma-Aldrich) in Tris-buffered saline $0.1 \%$ Tween 20 were applied and incubated overnight at $4^{\circ} \mathrm{C}$. Proteins were visualized by enhanced chemiluminescence (Chemiluminescence Reagent Plus, Perkin-Elmer Life Sciences, Waltham, MA) and the density was quantified using Kodak Imaging software, Kodak ID 3.5 (Perkin-Elmer Life Sciences). Membranes were stripped (Restore Western Blot Stripping Buffer, Thermo-Fisher Scientific, Scoresby, VIC, Australia) before being re-probed for total STAT3.

Cells were collected and RNA was extracted, transcribed, and PCR performed as previously described (Trenerry et al., 2011). The efficacy of TATA-box binding protein $(\boldsymbol{T B P})$ as an endogenous control was examined using the equation $2^{-\Delta \mathrm{Cq}}$. No change in the expression of this gene was observed. Primers were designed using NCBI/Primer BLAST and are outlined here or have been described previously (Caldow et al., 2011; Trenerry et al., 2011): CKM-Fwd: GGCATCTGGCACAATGACAA; CKM-Rev: GATGACCCGGAGGTGATCCT; MyoD-Fwd: CCGCCTGAGCAAAGTAAATGA; MyoD-Rev: GCAACCGCTGGTTTGGATT; TBP-Fwd: CGAATATAATCCCAAGCGGTTT; and TBP-Rev: CCGTGGTTCGTGGCTCTCT, where Fwd is forward and Rev is reverse.

Statistical analysis was performed using SPSS 15.0 (SPSS Inc., Chicago, IL). Unless stated otherwise, means were compared using an unpaired $t$-test. For pSTAT3/tSTAT3 measurements, a 2-way ANOVA was used and significant differences were determined using a Tukey post hoc test. Data are presented as mean \pm SEM.

Milk-derived proteins promote skeletal muscle protein synthesis and aid in muscle recovery (Crittenden et al., 2009). In vitro, milk-derived proteins have been shown to possess positive cross-species growth effects (Steimer et al., 1981). However, it is unknown which signaling pathways are responsible for these effects in human skeletal muscle. Because STAT3 is necessary for myoblast proliferation/differentiation (Sun et al., 2007; Wang et al., 2008), it is an appropriate target for measuring the effect of bovine-derived compounds on myogenesis. This study demonstrates that bovine- 


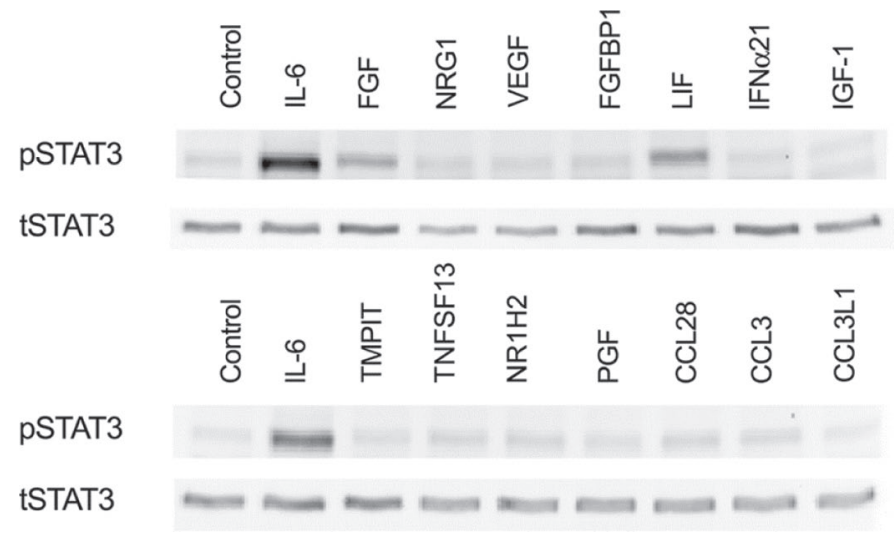

Figure 1. Signal transducer and activator of transcription-3 (STAT3) phosphorylation in human skeletal muscle cells following acute exposure to bovine-derived compounds. Representative immunoblots of protein extracted from differentiating human skeletal muscle cells exposed to a variety of bovine-derived compounds for $30 \mathrm{~min}$. The STAT3 Tyr705 phosphorylation increased in cells treated with fibroblast growth factor-1 (FGF) and leukemia inhibitory factor (LIF). pSTAT3 = phosphorylated STAT3; tSTAT3 = total STAT3; NRG1 = neuregulin 1; VEGF = vascular endothelial growth factor; FGFBP1 $=$ fibroblast growth factor binding protein $1 ;$ IFN $\alpha 21=$ interferon $\alpha$ 21: TMPIT $=$ transmembrane protein induced by TNF- $\alpha$; TNFSF13 $=$ tumor necrosis factor ligand superfamily, member 13; NR1H2 = nuclear receptor subfamily 1 , group $\mathrm{H}$, member 2 ; $\mathrm{PGF}=$ placental growth factor; CCL28 = chemokine (C-C motif) ligand 28; CCL3 = chemokine (C-C motif) ligand 3; CCL3L1 = chemokine (C-C motif) ligand 3 -like 1.

derived proteins LIF and FGF induced STAT3 phosphorylation in mature human skeletal muscle in vitro following a 30-min exposure (Figure 1), whereas other selected clones did not (Figure 1). However, it is possible that the amount of protein in these preparations was not sufficient to stimulate STAT3 phosphorylation or that the exposure time was not appropriate for all of the selected clones.

Following a 4-d treatment with FGF or LIF in proliferating or differentiating cells, STAT3 phosphorylation remained unchanged, despite elevated SOCS3 mRNA $(P<0.05$, Table 1$)$. However, STAT3 phosphorylation increased during differentiation when compared with proliferating cells $(P<0.05$, Table 1$)$. Interestingly, myoblast proliferation was reduced following a 4-d treatment with either bovine FGF or LIF. Reductions of 27 and $45 \%$ occurred in FGF- and LIF-treated cells, respectively $(P<0.05$, Table 1$)$, albeit without a change in apoptotic genes, indicating this may not be due to a reduction in cell viability (Table 1 ). When genes associated with differentiation were measured, MyoD and CKM increased expression following FGF treatment $(P<0.05$, Table 1$)$, as well as a trend for increased CKM expression in LIF-treated myoblasts $(P=0.06$, Table 1$)$. Despite a $70 \%$ increase following exposure to FGF and LIF, myogenin mRNA was not different (Table 1). Together these data indicate that a reduction in proliferation in the presence of bovinederived FGF or LIF may stimulate early maturation of human myoblasts. This is consistent with the action of recombinant acidic FGF (Düsterhöft and Pette, 1999) and LIF (Austin and Burgess, 1991) on proliferating murine muscle cells in vitro. However, recombinant

Table 1. Signal transducer and activator of transcription-3 (STAT3) phosphorylation, cellular proliferation, and mRNA expression data from proliferating and differentiating myoblasts ${ }^{1}$

\begin{tabular}{lccc}
\hline Item & Control & FGF & LIF \\
\hline Proliferation & \\
pSTAT3/tSTAT3 & & & \\
Cell proliferation $\left(\mathrm{Abs}^{3}\right.$; nm) & $0.068 \pm 0.027$ & $0.060 \pm 0.019$ & $0.066 \pm 0.009$ \\
Bcl-2 & $0.806 \pm 0.063$ & $0.638 \pm 0.062^{*}$ & $0.521 \pm 0.060^{* * *}$ \\
Bcl-XL & $0.034 \pm 0.009$ & $0.032 \pm 0.014$ & $0.025 \pm 0.003$ \\
Bax & $3.77 \pm 0.95$ & $4.68 \pm 0.39$ & $3.36 \pm 0.30$ \\
MyoD & $9.12 \pm 1.03$ & $14.08 \pm 2.28$ & $13.48 \pm 3.1$ \\
Myogenin & $9.95 \pm 1.51$ & $15.76 \pm 1.13^{*}$ & $13.43 \pm 1.5$ \\
CKM & $0.095 \pm 0.038$ & $0.165 \pm 0.019$ & $0.165 \pm 0.027$ \\
SOCS3 & $0.447 \pm 0.18$ & $0.636 \pm 0.069^{*}$ & $0.614 \pm 0.071$ \\
Differentiation & $5.01 \pm 0.62$ & $7.09 \pm 0.27^{*}$ & $8.39 \pm 0.71^{* *}$ \\
pSTAT3/tSTAT3 & & & \\
Myogenin & $0.132 \pm 0.005 \dagger$ & $0.132 \pm 0.024 \dagger$ & $0.143 \pm 0.017 \dagger$ \\
CKM & $1.13 \pm 0.22$ & $1.46 \pm 0.18$ & $0.955 \pm 0.118$ \\
SOCS3 & $11.32 \pm 1.65$ & $16.97 \pm 2.59$ & $9.65 \pm 1.98$ \\
D & $9.92 \pm 1.02$ & $8.29 \pm 1.32$ & $8.84 \pm 2.83$
\end{tabular}

${ }^{1}$ Data are arbitrary units (except where specified) presented as mean \pm SEM, representing the mean of 4 replicates.

${ }^{2}$ Phosphorylated (p)STAT3 was normalized to the abundance of total (t)STAT3. For mRNA expression, genes are normalized to TATA-box binding protein (TBP). FGF = fibroblast growth factor-1; LIF = leukemia inhibitory factor.

${ }^{3}$ Absorbance.

${ }^{*} P<0.05,{ }^{* * *} P<0.001$ : Significantly different from control cells.

$\dagger P<0.05$ : Significantly different from proliferating cells. 
FGF is traditionally thought to promote proliferation and prevent maturation of rodent muscle cell lines (Clegg et al., 1987; Sheehan and Allen, 1999; Uruno et al., 1999). It has been recently demonstrated that not all mechanisms regulating mouse satellite cell activation are conserved in human satellite cells (Bareja et al., 2014), highlighting the importance of performing studies using human primary cells. Only limited literature is available regarding the effect of FGF on human myoblasts, suggesting that human myoblasts respond differently to FGF compared with murine-derived cell lines.

In mature myotubes, these growth factors may not have any effect. Genes associated with differentiation were measured to determine the effect of chronic exposure to LIF and FGF in myotubes. No differences were observed for any of the genes measured for each treatment condition (Table 1). This may be attributable to the maturation stage of the myotubes.

In conclusion, bovine-derived proteins activate STAT3 in human skeletal muscle cells and may promote cellular maturation. Therefore, proteins found in the lactating mammary gland demonstrate biological activity in human cells in vitro. The significance of this finding to human health is unknown; however, it may be possible to generate enriched bovine protein fractions containing these peptides. Studies are required to probe bioavailability in response to oral ingestion and further elucidate their physiological effect.

\section{ACKNOWLEDGMENTS}

The authors thank Rani Watts (School of Exercise and Nutrition Sciences, Deakin University, Melbourne, Australia) for her assistance with the collection of cell culture samples following the peptide initial screen and Andrew Garnham (School of Exercise and Nutrition Sciences, Deakin University, Melbourne, Australia) for performing the muscle biopsies. This research was funded by Dairy Australia (\#DU12042). M. K. Caldow received a $\mathrm{PhD}$ scholarship from Dairy Australia; however, the funding body had no input in study design; in the collection, analysis, and interpretation of data; or in the writing of the report.

\section{REFERENCES}

Austin, L., and A. W. Burgess. 1991. Stimulation of myoblast proliferation in culture by leukaemia inhibitory factor and other cytokines. J. Neurol. Sci. 101:193-197.

Bareja, A., J. A. Holt, G. Luo, C. Chang, J. Lin, A. C. Hinken, J. M. Freudenberg, W. E. Kraus, W. J. Evans, and A. N. Billin.2014.
Human and mouse skeletal muscle stem cells: Convergent and divergent mechanisms of myogenesis. PLoS ONE 9:e90398.

Belford, D. A., M.-L. Rogers, G. O. Regester, G. L. Francis, G. W. Smithers, I. J. Liepe, I. K. Priebe, and F. J. Ballard. 1995. Milkderived growth factors as serum supplements for the growth of fibroblast and epithelial cells. In Vitro Cell. Dev. Biol. Anim. $31: 752-760$

Bergstrom, J. 1975. Percutaneous needle biopsy of skeletal muscle in physiological and clinical research. Scand. J. Clin. Lab. Invest. 35:609-616.

Brown, K. D., and D. M. Blakeley. 1984. Partial purification and characterization of a growth factor present in goat's colostrum. Similarities with platelet-derived growth factor. Biochem. J. 219:609-617.

Caldow, M. K., G. R. Steinberg, and D. Cameron-Smith. 2011. Impact of SOCS3 overexpression on human skeletal muscle development in vitro. Cytokine 55:104-109.

Clegg, C. H., T. A. Linkhart, B. B. Olwin, and S. D. Hauschka. 1987. Growth factor control of skeletal muscle differentiation: Commitment to terminal differentiation occurs in G1 phase and is repressed by fibroblast growth factor. J. Cell Biol. 105:949-956.

Crittenden, R., J. Buckley, D. Cameron-Smith, A. Brown, K. Thomas, S. Davey, and P. Hobman. 2009. Functional dairy protein supplements for elite athletes. Aust. J. Dairy Technol. 64:133-137.

Damerdji, O., F. Derouiche, C. Legrand, J. Capiaumont, J. M. Bour, M. Maugras, F. Belleville, P. Nabet, D. Paquet, and G. Linden. 1988. Utilization of whey fractions as a substitute for fetal calf serum in culture media. Biotechnol. Tech. 2:253-258.

Düsterhöft, S., and D. Pette. 1999. Evidence that acidic fibroblast growth factor promotes maturation of rat satellite-cell-derived myotubes in vitro. Differentiation 65:161-169.

Evans, W. J., S. D. Phinney, and V. R. Young. 1982. Suction applied to a muscle biopsy maximizes sample size. Med. Sci. Sports Exerc. 14:101-102.

Garofalo, R. P., and A. S. Goldman. 1998. Cytokines, chemokines, and colony-stimulating factors in human milk: The 1997 update. Biol. Neonate 74:134-142.

Grosvenor, C. E., and M. F. Picciano. 1993. Hormones and growth factors in milk. Endocr. Rev. 14:710-728.

Rayner, T. E., and A. J. Cowin. 2000. Mitogenic whey extract stimulates wound repair activity in vitro and promotes healing of rat incisional wounds. Am. J. Physiol. Regul. Integr. Comp. Physiol. 278:R1651-R1660.

Séverin, S., and X. Wenshui. 2005. Milk biologically active components as nutraceuticals: Review. Crit. Rev. Food Sci. Nutr. 45:645-656.

Sheehan, S. M., and R. E. Allen. 1999. Skeletal muscle satellite cell proliferation in response to members of the fibroblast growth factor family and hepatocyte growth factor. J. Cell. Physiol. 181:499-506.

Steimer, K. S., R. Packard, D. Holden, and M. Klagsbrun. 1981. The serum-free growth of cultured cells in bovine colostrum and in milk obtained later in the lactation period. J. Cell. Physiol. 109:223234

Sun, L., K. Ma, H. Wang, F. Xiao, Y. Gao, W. Zhang, K. Wang, X. Gao, N. Ip, and Z. Wu. 2007. JAK1-STAT1-STAT3, a key pathway promoting proliferation and preventing premature differentiation of myoblasts. J. Cell Biol. 179:129-138.

Trenerry, M. K., P. A. Della Gatta, and D. Cameron-Smith. 2011. JAK/STAT signaling and human in vitro myogenesis. BMC Physiol. 11:6.

Uruno, T., J. Oki, K. Ozawa, K. Miyakawa, H. Ueno, and T. Imamura. 1999. Distinct regulation of myoblast differentiation by intracellular and extracellular fibroblast growth factor-1. Growth Factors 17:93-113.

Wang, K., C. Wang, F. Xiao, H. Wang, and Z. Wu. 2008. JAK2/ STAT2/STAT3 are required for myogenic differentiation. J. Biol. Chem. 283:34029-34036. 